\title{
ARTHRODESIS OF THE TRAPEZIO-METACARPAL JOINT FOR OSTEOARTHRITIS
}

\author{
G. M. Müller, London, ENGLAND \\ From the Orthopaedic Unit, St Peter's Hospital, Chertsey
}

Painful osteoarthritis of the trapezio-metacarpal joint may result from occupations involving repetitive use of the thumb. Mild symptoms are common in middle-aged women, but advanced changes may be found in factory workers and particularly in those doing heavy sewing such as upholstery or mattress-making. In severe cases the thumb is held adducted and is used with caution; grasping, pinching and wringing movements are uncomfortable, and the lifting of heavy objects requiring a wide grip causes much pain.

Examination shows some swelling and local tenderness from synovial and capsular thickening. Abduction and extension movements are limited and opposition is carried out with difficulty. Thus for a pincer grip

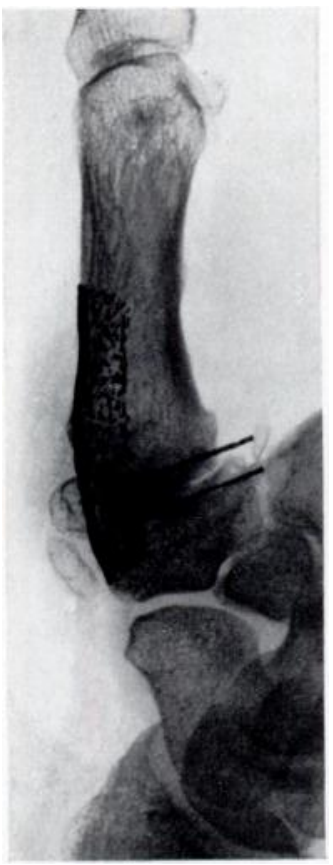

FIG. 1

To illustrate the technique of arthrodesis of the trapezio-metacarpal joint. the pulp of the thumb is applied to the distal joint of the index, whereas normally both tips meet to form an " $\mathrm{O}$ " in a position of great strength of the thumb. In addition to sclerosis of the subchondral bone and osteophytic lipping, radiographs often show a small detached osteophyte on the lateral aspect of the joint at the site of maximum tenderness.

Arthrodesis of the joint and excision of the trapezium (Gervis 1948) are two methods of dealing with the more severe cases. A sound arthrodesis retains the stability and power of the thumb, and the continuity of the line of bones from the radius through the scaphoid and trapezium to the first metacarpal being intact, the system is not deprived of one of its units of leverage. A case of "failed arthrodesis" can be treated by a second graft if symptoms continue, whereas grafting after "failed excision" of the trapezium must be difficult. One disadvantage is some difficulty in obtaining sound fusion because the subchondral bone of the trapezium is

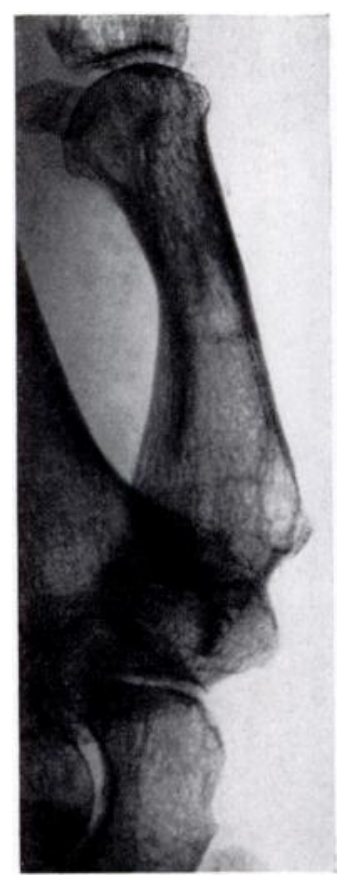

FIG. 2

To show sound arthrodesis with a bone graft from the ilium (Case 3).

sclerotic and there is little enough of the bone left after complete excision of the articular surface. Post-operative fixation must therefore be efficient and prolonged. Another disadvantage is the need for a separate incision over the tibia or ilium to obtain a bone graft of good quality. There is no doubt that a graft is essential for the reason that simple excision of the joint gives rise to fibrous ankylosis.

Technique of arthrodesis-The bones are exposed from the lateral subcutaneous aspect and the insertion of extensor ossis metacarpi pollicis is elevated. A narrow slot is cut in the first metacarpal and trapezium; in this way the alignment of the bones is not altered subsequently. The accessible capsule, the synovial membrane of the trapezio-metacarpal 
joint, and then the joint surfaces are excised; particular attention is paid to the medial part of the saddle-shaped surface of the trapezium which is difficult of access. The bones having been fitted together accurately, the graft is skidded into the slot and tightly impacted (Fig. 1). Source of the graft-In one case the oblong of metacarpal shaft raised from the slot was used as a sliding graft, but the quality of the bone was poor. Tibial grafts were used in four cases; one bilateral case operated upon by a colleague for the second side ended with a pseudarthrosis. The last three cases have had iliac grafts including some cortical bone cut with a finger chisel in one piece from the crest. A few cancellous chips have supplemented the main graft. The greater osteoblastic activity and elasticity of iliac bone make it superior to tibial bone for this purpose.

After-care-The thumb and wrist are fixed in a scaphoid type of plaster with the thumb in mid-opposition, and the limb is elevated for a few days. After two or three weeks a well-fitting cast is applied over stockinette and immobilisation is continued for a total of at least four months. When iliac bone is used even longer may be required despite the radiographic appearances. In two recent cases the plaster was removed after four months, when there was no tenderness locally or pain on springing the bones and when the radiographs seemed satisfactory, but within a week pain and tenderness recurred and fixation had to be resumed.

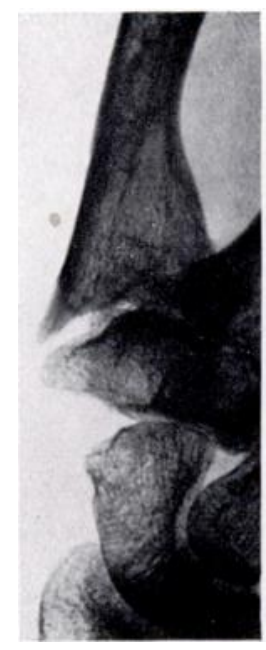

(ase 4. To show the range of opposition after operations resulting in fibrous ankylosis of the left trapezio-metacarpal joint and sound fusion of the right joint.

Results-Eight operations have been carried out on seven patients. Six joints fused soundly and gave excellent and painless function. One patient remains untraced. In one bilateral case for which tibial grafts were used, the right side gave a sound arthrodesis but the left side, operated on two years later, ended with fibrous ankylosis (Fig 3). At review four years after the second operation this patient said there was no difference in function between the two sides; she was working again as a draper's assistant, gripping heavy rolls of cloth between each thumb and forefinger with no loss of power.

\section{CASE REPORTS}

Case 1. Mrs F. S., aged fifty-eight years, housekeeper-Pain and swelling in right thumb for five months, increasing in severity. Arthrodesis with sliding metacarpal graft, March 1943. Plaster removed after four months; fusion solid. Good function subsequently.

VOL. $31 \mathrm{~B}$, No. 4 , NOVEMBER 1949 
Case 2. R. S., aged thirty-two years, tank mechanic-Blow on base of left thumb three years previously. Radiographs showed unusual prominence of the base of the first metacarpal and a distorted trapezium, with osteoarthritis in the carpo-metacarpal joint. Irthrodesis with tibial graft, August 1945. Plaster removed after five months; good function regained within a fortnight.

Case 3. Mrs H. S., aged fifty-eight years, factory worker-Pain in the joint since changing one type of work for another, both types involving repetitive thumb movements. Arthrodesis with iliac graft June 1948 (Fig. 2). Plaster removed after five months; early return of full function. Now able to touch the basal crease of the little finger with the tip of the thumb; free from pain and very pleased with the result.

Case 4. Mrs E. M., aged fifty-three years, draper's assistant--Long-standing pain in both thumbs, worse on the right side. Right side grafted in October 1943 with tibial bone. Plaster for five months and sound arthrodesis. Left side operated on in January 1945, again with a tibial graft. Pseudarthrosis occurred on this side. When seen in June 1948, the patient was pleased with both results and there was no difference in function or movement. She was able to oppose each thumb to the proximal phalanx of the little finger (Fig. 3).

Case 5. Mrs B. L., aged forty-two years, upholsterer-Severe symptoms in left thumb for two years Arthrodesis with iliac graft in May 1948. Plaster for five months; full painless movement soon after. Able to oppose tip of thumb to basal crease of little finger.

Case 6. Mrs A. C., aged fifty-one years, housewife-Arthrodesis with a tibial graft in December 1944 Patient cannot be traced.

Case 7. Mrs P. C., aged fifty-seven years, housewife-Symptoms in left thumb for two years. Arthrodesis in July 1947 with iliac bone. One year later, painless and excellent function.

Comment-Apart from the relief of pain given by the fusion the most striking feature of these cases is the early return of function after removal of the plaster cast. Arthrodesis of the trapezio-metacarpal joint scarcely affects the range of movement of the thumb, probably because of the compensatory increase in the range of the trapezio-carpal joints.

I am indebted to Mr B. H. Burns for permission to report five cases and for his stimulating interest; to $\mathrm{Mr} \mathrm{V}$. H. Ellis for a further case; and to Mr R. T. Whitley of the Institute of Orthopaedics for the printing of the radiographs.

\section{REFERENCE}

Gervis, W. H. (1948): Proceedings of the Royal Society of Medicine (Section of Orthopaedics), 40, 492. 